\title{
Neurosarcoidosis: Two case reports with multiple cranial nerve involvement and review of the literature
}

\author{
Milija Mijajlovica , Mihailo Mirkovic ${ }^{b}$, Violeta Mihailovic-Vucinic ${ }^{c}$, Vuk Aleksic $^{a},{ }^{\text {, Nadezda Covickovic-Sternic }}{ }^{\mathrm{a}}$
}

Background. Involvement of the central nervous system is registered in a relatively small number of patients with sarcoidosis. In this article we present two cases with various neurological symptoms that fulfill criteria for neurosarcoidosis (NS). In addition, we review the literature on NS with special attention to isolated cranial nerve involvement. Methods and Results. First patient: Neurological examination identified multiple cranial neuropathy, moderate rightsided hemiparesis, polyradiculoneuritis of the lower limbs and positive meningeal signs. Laboratory tests showed serum and cerebrospinal fluid (CSF) inflammatory abnormalities, with increased values of the angiotensin-converting enzyme (ACE). CSF analysis also showed presence of 9 oligoclonal lgG bands. Brain and spine magnetic resonance imaging (MRI) revealed diffuse meningopathy, and focal granulomatous lesion in the body of the L5 vertebra. Lung sarcoidosis was confirmed by additional diagnostic procedures. The patient was treated with Methylprednisolone and a tapering course of oral Prednisone, which reduced the pain in the back and legs and improved the strength of the right leg. However, the other neurological deficiencies remained. After confirming lung sarcoidosis, the patient received Methotrexate in addition to Prednisone but during the following 2 years the patient's condition progressively worsened and ended in death.

Second patient: Neurological findings showed weakness of the right $n$. oculomotorius and the right $n$. trochlearis, as well as the right-side face weakness. We found raised level of the ACE in serum and CSF. Thorax high-definition computed tomography (HDCTT) showed ribbon-like domains of discrete changes in the pulmonary parenchyma. MRI of the brain showed multiple white matter lesions. This patient also received Methylprednisolone followed by Prednisone, and after two months, ocular motility normalized.

Conclusion. The diagnosis of NS is always a challenge. For this rerason definitive diagnosis requires the exclusion of other causes of neuropathy. Multiple cranial neuropathies should always arouse suspicion of NS.

Key words: sarcoidosis, neurosarcoidosis, multiple cranial neuropathies

Received: November 24, 2012; Accepted with revision: June 6, 2013; Available online: June 27, 2013 http://dx.doi.org/10.5507/bp.2013.047

${ }^{a}$ Neurology Clinic, Clinical Center of Serbia and School of Medicine, University of Belgrade, Serbia ${ }^{b}$ Department of Neurology, Health Center Valjevo, Valjevo, Serbia

'Clinic for Pulmonary Diseases and Tuberculosis, Clinical Center of Serbia and School of Medicine, University of Belgrade, Serbia Corresponding author: Milija Mijajlovic, e-mail:milijamijajlovic@yahoo.com

\section{INTRODUCTION}

Sarcoidosis is a chronic systemic granulomatous disease of unknown etiology, which can only be diagnosed with certainty by histological examination. The prevalence of clinical involvement of the central and peripheral nervous system in sarcoidosis is estimated to $5-15 \%$, and very rarely it may selectively involve the nervous system ${ }^{1}$. Neurosarcoidosis (NS) is a diagnostic challenge, especially if systemic symptoms are absent, which happens in about $3 \%$ of patients with NS. Neurological symptoms are the primary manifestation of the disease in $62-74 \%$ of cases of NS (ref., ${ }^{2,3}$ ). Majority of patients with NS develop systemic symptoms after presenting neurological signs of disease.

\section{CASE REPORT 1}

A 45-year-old woman with a history of suspected cervical carcinoma underwent total hysterectomy when she was 34 years old, but likely, suspicion was never confirmed. When she was 43 , a regular oncological examination by chest radiography revealed a lesion in the apical part of the right lung. Because of that, thorax high-definition computed tomography (HDCTT) was performed, followed by fiber bronchoscopy. A malignant process was not identified. The patient was a smoker for many years and for several years she spoke in a hoarse voice. She also suffered from pneumonia a few times.

The patient's father died of lung cancer at the age of 70 and the patient's grandfather suffered from lung tuberculosis.

First neurological difficulties manifested at the age of 37 when she was hospitalized at the Neurology Department of the Health Centre Valjevo. At that time, the moderate right-sided hemiparesis was identified, so we first performed computed tomography (CT) scan of the brain, which showed normal findings.

At the age of 45 she felt numbness of the feet and pain in the back, which gradually spread to the legs. In the course of one year the patient noticed weakness of dorsi- 


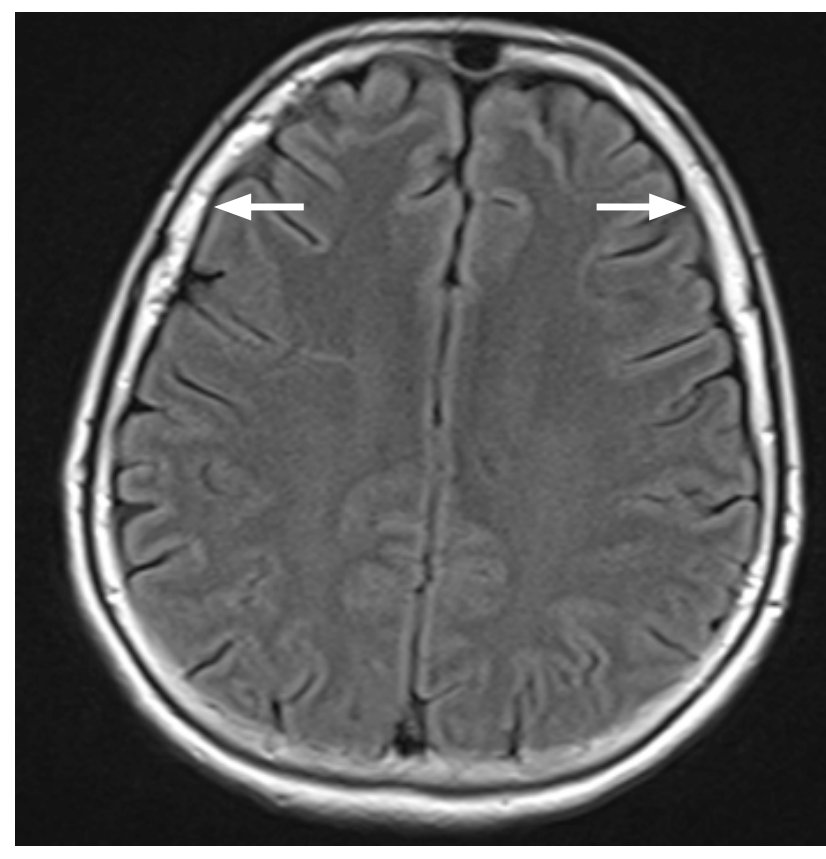

Fig. 1. MRI scan of the brain (fluid attenuated inversion recovery-FLAIR sequence): diffuse meningopathy.

flexion of the left foot, unstable walking, as well as certain difficulty while climbing the stairs. During that period she gradually developed slightly reduced visual acuity on the left eye, sensory loss and weakness in the left side of face followed by loss of taste over the anterior two-thirds of the left side of the tongue and reduced lacrimation on the left eye, left-sided hearing loss, swallowing difficulties and numbness in the left side of the tongue. As associated symptoms and signs, intermittent sub febrile temperature, fatigue, weight loss and palm rash, were recorded-

Laboratory tests showed serum and CSF inflammatory abnormalities, with increased values of the angiotensin-converting enzyme (ACE), but both were negative on infectious diseases. CSF analysis also showed presence of 9 oligoclonal IgG bands. Brain and spine MRI revealed diffuse meningopathy (Fig. 1, 2), and focal granulomatous lesion in the body of the L5 vertebra (Fig. 3). Chest radiographs, HDCTT and bronchoscopy with transbronchial biopsy confirmed lung sarcoidosis. Bone scintigraphy showed normal findings.

General examination showed only inguinal and axillary lymphadenopathy. Neurological findings identified multiple cranial neuropathy (II, V, VII, VIII, IX, XI and XII nerves), moderate right-sided weakness, polyradiculoneuritis of the lower limbs and positive meningeal signs.

Following these neurological findings, further examination focused on neurosarcodiosis, so the patient was hospitalized at the Neurology Department of the Health Care Centre Valjevo for the second time. Laboratory tests yielded: white blood cells count $-11.300 / \mathrm{mm} 3$, ACE in serum and CSF were 71.5 U/L and 2.0 U/L (0-0), respectively. CSF analysis indicated lymphocytic pleocytosis (10 cells), raised protein level $(0.77 \mathrm{~g} / \mathrm{L})$, hypoglycorrhachia $(2.3 \mathrm{mmol} / \mathrm{L})$ and the presence of 9 oligoclonal $\mathrm{IgG}$ bands detected by CSF electrophoresis. Cultures for my-

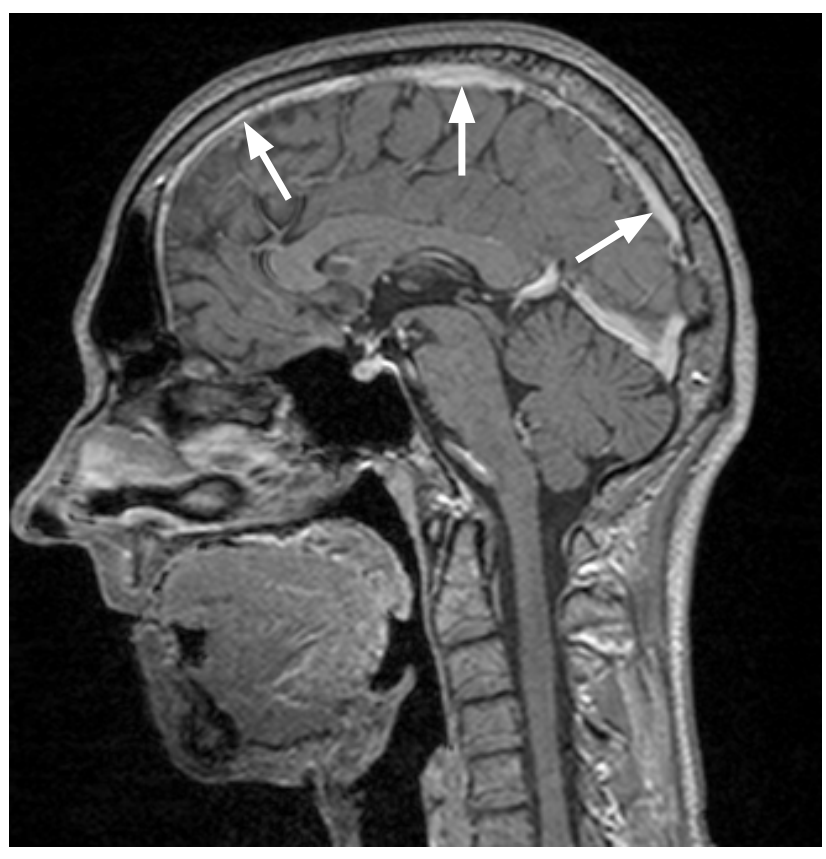

Fig. 2. MRI scan of the brain (T1 weighted sequence): diffuse meningopathy.

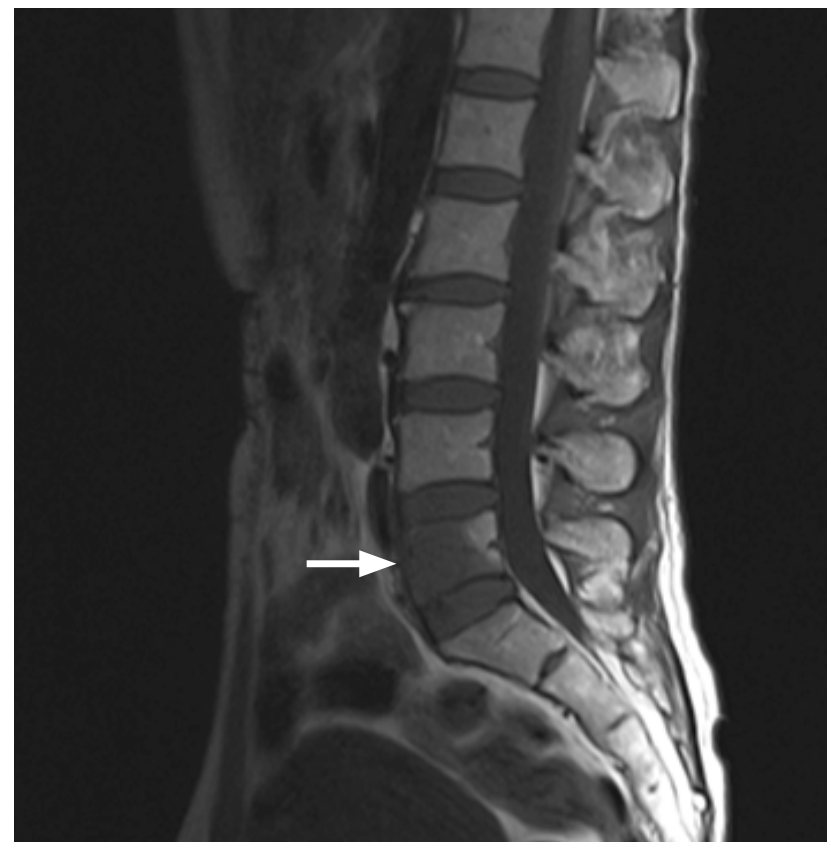

Fig. 3. MRI scan of the spinal column (T1 weighted sequence): focal granulomatous lesion in the body of the L5 vertebra.

cobacterium and Mantoux test were negative and malignant cells in CSF were not identified.

Other causes of granulomatous diseases, such as infections (cryptococcus, histoplasma, toxoplasma, treponema, and Whipple's disease), inflammatory disorders (Wegener's granulomatosis, giant cell arteritis, systemic lupus erythematosus, and Churg-Strauss syndrome) were excluded by additional analyses. Influence of chemicals, radiotherapy and chemotherapy was also excluded. 
Chest radiography showed a change in the upper part of the right lung. HDCTT showed parenchymal tissue changes in the right lung, along with hilar and mediastinal lymph node enlargement. There was a progression of the changes compared to the initial findings.

The patient was treated with Methylprednisolone (1000 mg/day for three days) and a tapering course of oral Prednisone, which reduced the pain in the back and legs and improved the strength of the right leg. However, the rest of the neurological deficiency remained.

The patient was then referred to the Neurology Clinic in Belgrade for additional tests as NS was suspected. The laboratory tests conducted there showed ACE in the serum 47.2 U/L and CSF 4.3 U/L (0-0), respectively. Tumor markers Cyfra 21-1, NSE, AFP, CA 15-3 and CA 19-9 were within referential value range, while CA 125 and CEA were high.

CSF analysis revealed 3 lymphocytes and 164 erythrocytes, raised protein level $(0.95 \mathrm{~g} / \mathrm{L})$, hypoglycorrhachia $(1.8 \mathrm{mmol} / \mathrm{L})$, and the presence of 9 oligoclonal $\mathrm{IgG}$ bands, detected by electrophoresis.

Lower limbs electromyoneurography (EMNG) test revealed a strong and chronic lesion of the S1 and L5 root on the right side, while on the left side there was a strong subacute lesion of the L5 and a mild lesion of the L4 root.

Transbronchial biopsy isolated epithelioid granulomatous foci and confirmed lung sarcoidosis. Therefore, the patient was transferred to the Institute for Pulmonary Diseases in Belgrade, where therapy included Methotrexate in addition to Prednisone, but during next 2 years the patient's condition progressively worsened and ended in patient's death.

\section{CASE REPORT 2}

A 70-year-old man was admitted at the Neurology Clinic of the Clinical Center of Serbia because of the right-sided ptosis and predominant vertical double vision, worse on downward gaze, the most pronounced during attempted medial gaze. Seven years before, at the age of 63 , he was treated for peripheral weakness of the right side of the face designated as Bell's palsy.
Past medical history showed slightly higher glucose blood level and elevated blood pressure as well as irregular heartbeats i.e. premature ventricular contractions (PVC).

Neurological findings showed involvement of the right n. oculmotorius, severe right $\mathrm{n}$. trochlearis palsy and the peripheral weakness of the right side of the face with synkinetic movements of the right corner of the mouth during eye squeezing (as the late sequelae of Bell's palsy). Other neurological findings were normal.

The ACE level in serum was $102.3 \mathrm{U} / \mathrm{L}$ (8-52), and in CSF 3.9 U/L (0-0), respectively. CSF analysis showed normal protein level, normal glycorrhachy, 1 lymphocyte, and no presence of oligoclonal IgG bands. Cultures for mycobacterium and Mantoux test were negative, while the cytological examination of CSF did not reveal any malignant cells. Additional examination excluded other causes of granulomatous diseases. Prostigmine test was negative.

MRI of the brain showed multiple white matter, T2weighted and fluid attenuated inversion recovery hyperintense lesions, in the periventricular areas. HDCTT showed ribbon-like domains of discrete changes in pulmonary parenchyma, but with regular mediastinum configuration and with no signs of lymphadenomegaly. Cerebral Magnetic Resonance Angiography (MRA) revealed no stenosis, aneurysm or arteriovenous malformations, but only slightly reduced terminal branches of both middle cerebral arteries. Twenty-four hour holter electrocardiographic monitoring was performed, but no PVCs were found. Structural heart diseases were ruled out by conventional cardiac examinations.

The patient was treated with Methylprednisolone ( $1000 \mathrm{mg} /$ day for three days), followed by $60 \mathrm{mg}$ of Prednisone a day. The patient was monitored for two months, after which double vision disappeared and bulbous motility was normal.

\section{DISCUSSION}

Sarcoidosis is an inflammatory granulomatous disease affecting multiple organ systems. NS is characterized by central nervous system involvement and even though is a less common manifestation of sarcoidosis, its symptoms

Table 1. Diagnostic criteria for neurosarcoidosis ${ }^{2}$.

Proposed criteria for the diagnosis of neurosarcoidosis

\begin{tabular}{ll}
\hline Definite & - clinical presentation suggestive of neurosarcoidosis \\
Probable & - positive nervous system histology \\
& - clinical syndrome suggestive of neurosarcoidosis \\
& laboratory support for CNS inflammation (elevated levels of CSF protein and/or cells, the presence of \\
& - eligoclonal bands and/or MRI evidence compatible with neurosarcoidosis) \\
& - indirect indicators from Gallium scan, chest imaging and serum ACE) \\
& - exclusion of alternative diagnoses \\
Possible & - clinical presentation suggestive of neurosarcoidosis \\
& -
\end{tabular}


can be devastating and may present to the physician in a number of guises.

Reviewing 68 cases of NS, Zajicek et al. formulated a series of diagnostic criteria for NS, which are presented in Table 1 (ref. $\left.^{2}\right)$. On the basis of these criteria, the diagnosis of NS can be: definite, probable or possible. Here we report two patients, the first one with probable diagnosis (clinical syndrome suggestive of neurosarcoidosis with laboratory support for CNS inflammation and exclusion of alternative diagnoses) and the second one with possible diagnosis (clinical presentation suggestive of neurosarcoidosis with exclusion of alternative diagnoses) of NS.

The clinical course may be acute, subacute or chronic with insidious onset. Systemic inflammatory indicators are altered only occasionally in NS.

Clinical symptoms of intracranial neurosarcoidosis depend on the location of lesions. Meninges of the skull base, hypothalamus and pituitary gland are the most common sites ${ }^{4-12}$.

The most common neurological symptoms are cranial nerve deficits, headache and seizures ${ }^{2,7,10-17}$. The facial nerve is most frequently affected, either due to a meningeal reaction or secondary to inflammation in the parotid gland. Facial palsies may occur unilaterally and simultaneously or sequentially bilaterally ${ }^{2,18}$. With exception of paresis of the VII cranial nerve, due to proximate inflammation of the parotid gland, nervous system involvement is not prone to spontaneous remission ${ }^{19}$. Both patients described here suffered from peripheral facial weakness, which did not improve in our first patient, while the second patient manifested incomplete resolution of peripheral facial weakness, after the treatment with corticosteroids.

In addition, syndromes deriving from the involvement of virtually every other cranial nerve have been reported ${ }^{2}$. In addition to the left facial nerve palsy, our first patient manifested damage of the left optic, trigeminal, auditory, hypoglossal, and the right accessory nerve, while in the second patient involvement of the right oculomotor and trochlear nerve was determined.

Meningeal symptoms may be acute or chronic. Symptoms and signs include fever, headache, neck rigidity, and sterile CSF with pleocytosis (particularly lymphocytes) $\left(\right.$ ref. $\left.^{20}\right)$. Sometimes mental status changes and polyradiculopathy could be present ${ }^{21,22}$. Our first patient suffered from poliradiculopathy and there were positive meningeal signs.

NS is difficult to diagnose, partly because the neurological presentation is polymorphic and nonspecific and it should be suspected after ruling out other inflammatory diseases, including cases of cryptogenic subacute meningo-myelopathies, especially when they respond to corticosteroids but tend to recur after suspending the drug. Hence, differential diagnosis includes chronic and subacute meningitis, multiple sclerosis, myelopathies and tumour-like lesions ${ }^{23-25}$. In both cases we performed extensive examination in order to exclude other differential diagnostic possibilities.

ACE levels are raised in $50 \%$ of patients with neurosarcoidosis (range 35-75\%). Some authors regard ACE levels in CSF as being useful in both the diagnosis and follow-up of NS (ref. ${ }^{26,27}$ ). However, many authorities now regard this test as non-specific and insensitive. CSF ACE may be raised in infections and malignancy, and appears unhelpful in any therapeutic decision making ${ }^{28}$. This is illustrated by one study of 32 patients with sarcoidosis, including 20 with NS: CSF ACE levels were raised in only 55\% of patients with NS, in $5 \%$ of patients with sarcoidosis not apparently involving the nervous system, and in 13\% of patients with other neurological diseases ${ }^{29}$. In both of our patients levels of ACE in serum and CFS were raised.

Hypercalcaemia is often seen in pulmonary sarcoidosis (a result of additional ahydroxylation occurring in the sarcoid lesions in the lung), while many cases of NS are reported with completely normal calcium levels, and similarly the erythrocyte sedimentation rate is raised only in a minority of cases. Both blood tests were normal in our presented patients.

The diagnostic value of chest radiography in suspected systemic sarcoidosis is undisputable: abnormalities are seen in $90 \%$, but of course, a normal chest X-ray does not exclude generalized sarcoidosis ${ }^{30}$. HDCTT is useful in diagnosing pulmonary sarcoidosis and also assists in targeting transbronchial biopsy. It is approximately $89 \%$ and $98 \%$ sensitive for hilar and mediastinal lymphadenopathy respectively, and $23 \%$ sensitive for pulmonary infiltrates ${ }^{31}$. Both diagnostic methods were positive in our first patient.

CSF abnormalities in NS are usually nonspecific, varying and they change over time and include mild pleocytosis, high protein content, and sometimes slightly lowered glucose concentrations (in about a fifth of patients) (ref. ${ }^{32}$ ). These changes may be marked and dictate differential diagnosis with tuberculous meningitis, or exhibit oligoclonal bands and orient diagnosis towards a demyelinating disease. Furthermore, increases in the concentration of ACE (ref. ${ }^{26,27,33-37}$ ), IgG-index ${ }^{38-40}$, oligoclonal bands ${ }^{38,39,41}$, CD4/CD8 lymphocyte ratios ${ }^{42}$, lysosymes and beta 2-microglobulin concentrations ${ }^{43}$ in CSF have been reported. Most studies report evidence of intrathecal synthesis of immunoglobulins with oligoclonal bands in about $30-40 \%$ of patients with NS (ref. ${ }^{2}$ ) while others suggest that this is uncommon ${ }^{38}$. CSF showed signs of inflammation accompanied with intrathecal synthesis of immunoglobulins with oligoclonal bands in our first patient.

About a third of patients with NS have normal CSF (ref. ${ }^{31,44,45}$ ), which was the case with our second patient, while Marangoni at al. claim that CSF analysis may be normal, but only after cycles of treatment with corticosteroids and/or immunosuppressants ${ }^{46}$. A number of elements may help to differentiate NS from tuberculous meningitis: failure to identify bacteria, negative cultures and polymerase chain reactions in 3 consecutive samples, persistently negative tuberculin tests, less aggressive clinical course, failure to respond to anti-tuberculous therapy. Moreover, in disorders such as multiple sclerosis and systemic lupus erythematosus, similar CSF abnormalities could be found.

MRI is also far superior in defining parenchymal involvement, with a reasonable level of sensitivity for intracranial abnormalities (up to $82 \%$ ) but poor specificity, 
with a wide spectrum of imaging findings ${ }^{47,48}$. About $40 \%$ of patients with NS have either leptomeningeal enhancement with gadolinium (present in the first patient), or multiple white matter lesions in the periventricular areas (present in our second patient) that may be difficult to distinguish from those seen in multiple sclerosis ${ }^{49}$. The unenhanced MRI is unsatisfactory because of the artifacts near bone, CSF and meninges on both T1 and T2weighted images. Tuberculosis, other bacterial, fungal, and neoplastic processes such as meningeal carcinoma, lymphoma and leukemia, may also enhance with gadolinium and mimic sarcoid radiographically. The MRI lesions in NS are more diffuse and smaller than those usually seen in tuberculous meningitis ${ }^{46}$. The low diagnostic yield for MRI in some series may reflect the effect of therapy because the MRI changes can disappear with successful treatment of the disease.

The diagnostic procedure should always aim to locate a site of disease that is accessible to biopsy. HDCTT, broncheoalveolar lavage (BAL) and contrast enhanced MRI scans are valuable means of suspecting the diagnosis and authorizing immunosuppressant therapy, when diagnostic biopsy is not possible.

In the absence of positive histology, the diagnosis may be supported by other types of investigation, such as BAL with analysis of the lymphocyte subpopulations. A prevalence of CD4 lymphocytes on BAL, with a CD4:CD8 ratio higher than 3.5 , has a positive predictive value of $76 \%$, with $53 \%$ sensitivity and $94 \%$ specificity $^{50}$.

The prognosis may be negatively influenced by various factors, including genetic factors and onset after 40 years of age $\mathrm{e}^{51}$. Sarcoidosis has a variable natural course: about $30 \%$ of cases show spontaneous remission, while disorders become chronic in $10-30 \%$ of cases $^{52}$. One to five percent of patients die, mainly from respiratory insufficiency, myocardiopathy or CNS lesions. In fact, disability and mortality rates are high in patients with CNS involvement $^{3,53}$.

Conventional treatment of pulmonary sarcoidosis is based on oral corticosteroids, but no studies have been published that define optimum dose or duration ${ }^{54}$. Hence, therapy is generally adjusted to patient response. Most patients affected by NS responded to corticosteroids only partially, prompting the need to associate an immunosuppressant drug ${ }^{3,55,56}$. Our first patient manifested a partial improvement caused by corticosteroid therapy, so that Methotrexate was added to the treatment, while the second patient responded well to corticosteroids.

Establishing a diagnosis of NS is therapeutically essential, since corticosteroid and immunosuppressant treatment must be started and continued for years in order to prevent, or at least limit, otherwise permanent disability. Early drug reduction led to a return of symptoms in all described cases. Neurological impairment results not only from florid inflammation, but also from progression of the fibrosis, which entraps the roots, compresses and deforms the spinal cord profile and brain base structures. This late stage of the disease may also benefit from immunosuppressant therapy.

\section{ABBREVIATIONS}

ACE, angiotensin-converting enzyme; BAL, broncheoalveolar lavage; CSF, cerebrospinal fluid; CT, computed tomography; EMNG, electromyoneurography test; ESR, erythrocyte sedimentation rate; HDCTT, thorax high-definition computed tomography; MRA, magnetic resonance angiography; MRI, magnetic resonance imaging; NS, neurosarcoidosis; PVC, premature ventricular contractions.

\section{AKNOWLEDGEMENT}

This work was granted by the Ministry of Education and Science of the Republic of Serbia. (Project No. 175022).

Authorship contributions: All authors equally contributed to preparing the manuscript.

Conflict of interest statement: None declared.

\section{REFERENCES}

1. Hoitsma E, Faber CG, Drent M, Sharma OP. Neurosarcoidosis: a clinical dilemma. Lancet Neurol 2004;3:3.

2. Zajicek JP, Scolding NJ, Foster O, Rovaris M, Evanson J, Moseley IF, Scadding JW, Thompson EJ, Chamoun V, Miller DH, McDonald WI, Mitchell D. Central nervous system sarcoidosis - diagnosis and management. Q J Med 1999;92:103-17.

3. Ferreby D, de Seze J, Stojkovic T, Hachulla E, Wallaert B, Destée A, Hatron PY, Vermersch P. Long-term followup of neurosarcoidosis. Neurology 2001;57:927-9.

4. Brooks ML, Wang AM, Black PM, Haikal N. Subdural mass lesion secondary to sarcoid granuloma. MR and CT findings and differential diagnosis. Comput Med Imaging Graph 1989;13:199-205.

5. Bullmann C, Faust M, Hoffmann A, Heppner C, Jockenhovel F, MüllerWieland D, Krone W. Five cases with central diabetes insipidus and hypogonadism as first presentation of neurosarcoidosis. Eur J Endocrinol 2000;142:365-72.

6. Christoforidis GA, Spickler EM, Recio MV, Metha BM. MR of CNS sarcoidosis: correlation of imaging features to clinical symptoms and response to treatment. AJNR Am J Neuroradiol 1999;20:655-9.

7. Delaney P. Neurological manifestations in sarcoidosis: review of the literature, with report of 23 cases. Ann Intern Med 1977;87:336-45.

8. Lexa FJ, Grossman RI. MR of sarcoidosis in the head and spine: spectrum of manifestation and radiographic response to steroid therapy. AJNR Am J Neuroradiol 1994;15:973-82.

9. Sharma OP, Anders A. Neurosarcoidosis. A report of ten patients illustrating some usual and unusual manifestations. Sarcoidosis 1985;2:96-106.

10. Ricker W, Clark M. Sarcoidosis: a clinical pathologic review of 300 cases. Am J Clin Pathol 1949;19:725-49.

11. Scott TF. Neurosarcoidosis: progress and clinical aspects. Neurology 1993;43:8-12.

12. Sharma OP, Sharma AM. Sarcoidosis of the nervous system: a clinical approach. Arch Intern Med 1991;151:1317-21.

13. Chen RC, McLeod JG. Neurological complications of sarcoidosis. Clin Exp Neurol 1989;26:99-112.

14. Luke RA, Stern BJ, Krumholz A, Johns CJ. Neurosarcoidosis: the longterm clinical course. Neurology 1987;37:461-3.

15. Pickuth D, Spielmann RP, Heywang-Kobrunner SH. Role of radiology in the diagnosis of neurosarcoidosis. Eur Radiol 2000;10:941-4.

16. Sharma OP. Neurosarcoidosis: a personal perspective based on the study of 37 patients. Chest 1997;112:220-8.

17. Stern BJ, Krumholz A, Johns C, Scott P, Nissim J. Sarcoidosis and its neurological manifestations. Arch Neurol 1985;42:909-17. 
18. Newman LS, Rose CS, Meier LA. Review article. Sarcoidosis. N Engl J Med 1997;336:1224-34.

19. Nowak DA, Widenka DC. Neurosarcoidosis: a review of its intracranial manifestation. J Neurol 2001;248:363-72.

20. Plotkin GR, Patel BR. Neurosarcoidosis presenting as chronic lymphocytic meningitis. Pa Med 1986;89:36-7.

21. Mayer SA, Yim GK, Onesti ST, Lynch T, Faust PL, Marder K. Biopsyproven isolated sarcoid meningitis. J Neurosurg 1993;78:994-6.

22. Jensen S, Jensen IW. Bilateral papilledema with normal CT-scan in neurosarcoidosis. Acta Med Scand 1987;222:381-3.

23. Kelly RB, Mahoney PD, Cawley KM. MR demonstration of spinal cord sarcoidosis: report of a case. AJNR Am J Neuroradiol 1988;9:197-9.

24. Jaster JH, Dohan FC Jr, Bertorini TE, Bass JE, Mönkemüller KE, Handorf CR, Gerald BE. Solitary spinal cord sarcoidosis without other manifestations of systemic sarcoidosis. Clin Imaging 1997;21:17-22.

25. Levivier M, Brotchi J, Balériaux D, Pirotte B, Flament-Durand J. Sarcoidosis presenting as an isolated intramedullary tumor Neurosurgery 1991;29:271-6.

26. Oksanen V, Fyhrquist F, Somer H, Grönhagen-Riska C. Angiotensin converting enzyme in cerebrospinal fluid: a new assay. Neurology 1985;35:1220-3.

27. Tahmoush AJ, Amir MS, Connor WW, Farry JK, Didato S, Ulhoa-Cintra A, Vasas JM, Schwartzman RJ, Israel HL, Patrick H. CSF-ACE activity in probable CNS neurosarcoidosis. Sarcoidosis Vasc Diffuse Lung Dis 2002;19:191-7.

28. Dale J. O'Brien JF. Determination of angiotensin-converting enzyme levels in cerebrospinal fluid is not a useful test for the diagnosis of neurosarcoidosis. Mayo Clin Proc 1999;74:535

29. Oksanen V. New cerebrospinal fluid, neurophysiological and neuroradiological examinations in the diagnosis and follow-up of neurosarcoidosis. Sarcoidosis 1987;4:105-10

30. Oksanen V. Neurosarcoidosis: clinical presentations and course in 50 patients. Acta Neurol Scand 1986;73:283-90.

31. Nuria A, Reis A, Bernardo J, Antunes P, Segorbe A, Eugenio L. Mediastinoscopy in the diagnose of sarcoidosis. Rev Port Pneumo 2003;9(5):36-7.

32. Powers WJ, Miller FM. Sarcoidosis mimicking glioma: case report and review of intracranial sarcoidosis like mass lesions. Neurology 1981:31:907-10.

33. Jones DB, Mitchell D, Horn DB, Edwards CR. Cerebrospinal fluid an giotensin converting enzyme levels in the diagnosis of neurosarcoidosis. Scott Med J 1991;36:144-5.

34. Rubinstein I, Hoffstein V. Angiotensin-converting enzyme in neurosarcoidosis. Arch Neurol 1987;44:249-50.

35. Oksanen V, Fyhrquist F, Gronhagen-Riska C, Somer H. CSF angiotensin-converting enzyme in neurosarcoidosis. Lancet 1985;1:1050-1.

36. Chan Seem CP, Norfolk G, Spokes EG. CSF angiotensin-converting enzyme in neurosarcoidosis. Lancet 1985;1:456-7.

37. Schweisfurth $H$, Schioberg-Schiegnitz S, Kuhn W, Parusel B.
Angiotensin I converting enzyme in cerebrospinal fluid of patients with neurological diseases. Klin Wochenschr 1987;65:955-8.

38. Borucki SJ, Nguyen BV, Ladoulis CT, McKendall RR. Cerebrospinal fluid immunoglobulin abnormalities in neurosarcoidosis. Arch Neurol 1989;46:270-3.

39. McLean BN, Mitchell DN, Thompson EJ. Local synthesis of specific IgG in the cerebrospinal fluid of patients with neurosarcoidosis detected by antigen immunoblotting using Kveim material. J Neurol Sci 1990;99:165-75.

40. Scott TF, Seay AR, Goust JM. Pattern and concentration of IgG in cerebrospinal fluid in neurosarcoidosis. Neurology 1989;39:1637-9.

41. Oksanen V. Neurosarcoidosis. Sarcoidosis 1994 11:76-9.

42. Juozevicius JL, Rynes RI. Increased helper/suppressor T-lymphocyte ratio in the cerebrospinal fluid of a patient with neurosarcoidosis. Ann Intern Med 1986:104:807-8.

43. Oksanen V, Gronhagen-Riska C, Tikanoja S, Somer H, Fyhrquist F. Cerebrospinal fluid lysozyme and beta 2-microglobulin in neurosarcoidosis. J Neurol Sci 1986;73:79-87.

44. Sharma OP. Neurosarcoidosis. Chest 1991;100:301-2.

45. Lynch JP 3rd. Neurosarcoidosis: how good are the diagnostic tests? J Neuroophthalmol 2003:23:187-9.

46. Marangoni S, Argentiero V, Tavolato B. Neurosarcoidosis: Clinical description of 7 cases with a proposal for a new diagnostic strategy. J Neurol 2006;253:488-95.

47. Pickuth D, Heywang-Kobrunner SH. Neurosarcoidosis: evaluation with MRI. J Neuroradiol 2000;27:185-8.

48. Mañá J. Magnetic resonance imaging and nuclear imaging in sarcoidosis. Curr Opin Pulm Med 2002;8:457-63.

49. Miller DH, Kendall BE, Barter S, Johnson G, MacManus DG, Logsdail $\mathrm{SJ}$, Ormerod IE, McDonald WI. Magnetic resonance imaging in central nervous system sarcoidosis. Neurology 1988;38:378-83.

50. Costabel U, Zaiss AW, Guzman J. Sensitivity and specificity of BAL findings in sarcoidosis. Sarcoidosis 1992;9(Suppl 1):211-4.

51. Mañá J, Salazar A, Manresa F. Clinical factors predicting persistence of activity in sarcoidosis: a multivariate analysis of 193 cases. Respiration 1994;61:219-25.

52. Hunninghake GW, Costabel U, Ando M, Baughman R, Cordier JF, du Bois R, Eklund A, Kitaichi M, Lynch J, Rizzato G, Rose C, Selroos O, Semenzato G, Sharma OP. ATS/ERS/WASOG Statement on Sarcoidosis. Sarcoidosis vasculitis and diffuse lung diseases 1999:16:149-73.

53. Chapelon-Abric C, Ziza JM, Piette JC, Blétry O, Wechsler B, Bousser MG, Godeau P. Neurosarcoidosis. Ann Med Intern 1991;142:601-8.

54. Paramothayan S, Jones PW. Corticosteroid therapy in pulmonary sarcoidosis. JAMA 2002:287:1301-7.

55. Agbogu BN, Stern BJ, Sewell C, Yang G. Therapeutic considerations in patients with refractory neurosarcoidosis. Arch Neurol 1995;52:8759.

56. Lower EE, Broderick JP, Brott TG, Baughman RP. Diagnosis and management of neurological sarcoidosis. Arch Intern Med 1997;157:1864-8. 